\title{
Scale-dependent Response by Breeding Songbirds to Residential Development Along Lake Superior
}

\author{
Author(s): Michelle T. Ford and David J. Flaspohler \\ Source: The Wilson Journal of Ornithology, 122(2):296-306. \\ Published By: The Wilson Ornithological Society \\ DOI: http://dx.doi.org/10.1676/09-137.1 \\ URL: http://www.bioone.org/doi/full/10.1676/09-137.1
}

BioOne (www.bioone.org) is a nonprofit, online aggregation of core research in the biological, ecological, and environmental sciences. BioOne provides a sustainable online platform for over 170 journals and books published by nonprofit societies, associations, museums, institutions, and presses.

Your use of this PDF, the BioOne Web site, and all posted and associated content indicates your acceptance of BioOne's Terms of Use, available at www.bioone.org/page/ terms of use.

Usage of BioOne content is strictly limited to personal, educational, and non-commercial use. Commercial inquiries or rights and permissions requests should be directed to the individual publisher as copyright holder. 


\title{
SCALE-DEPENDENT RESPONSE BY BREEDING SONGBIRDS TO RESIDENTIAL DEVELOPMENT ALONG LAKE SUPERIOR
}

\author{
MICHELLE T. FORD ${ }^{1,2,3}$ AND DAVID J. FLASPOHLER ${ }^{1}$
}

\begin{abstract}
We examined the influence of shoreline residential development on breeding bird communities along forested portions of Lake Superior and hypothesized that anthropogenic changes related to housing development would alter bird community structure compared to areas without human development. We used point counts to compare relative abundance of bird species in relation to residential development at coarse (along $1 \mathrm{~km}$ shoreline stretches with and without housing/cottage development) and fine (developed and undeveloped sides of a shoreline access road) spatial scales during the 2005 breeding season. More species had development related differences in abundance at the finer-scale analysis than at the coarse scale. American Crows (Corvus brachyrhynchos) and American Robins (Turdus migratorius) were more abundant on the developed, shoreline side of shoreline access roads. Red-breasted Nuthatches (Sitta canadensis), Blackthroated Green Warblers (Dendroica virens), and Red-eyed Vireos (Vireo olivaceus) were more abundant on the undeveloped, inland side of shoreline access roads. Several species were detected exclusively in developed or undeveloped forest areas. The pattern of development-related differences in relative abundance of bird species depended on the scale at which data were analyzed, suggesting that many species may respond to habitat differences within the $100 \mathrm{~m}$ scale quite distinct from how they respond to differences at the scale of thousands of meters. Received 27 August 2009. Accepted 23 December 2009.
\end{abstract}

Rural population growth in many parts of the United States has increased in the last 15 years (Long and Nucci 1997, Gustafson et al. 2005). Growth in parts of the northern Great Lakes Region has been concentrated around inland lakes (Radeloff et al. 2001, Gonzalez-Abraham et al. 2006). Two-thirds of previously undeveloped inland lakes in northern Wisconsin (i.e., lakes with no residential housing) have become developed with homes and cottages near the shoreline since 1965 (Lindsay et al. 2002, Elias and Meyer 2003). Housing development alters shoreline habitat through changes in habitat structure and plant species composition, which can create discontinuities in plant communities resulting in fragmentation (Brown 2003). These changes have the potential to influence many taxa including forest birds (Vale and Vale 1976, Mills et al. 1989, Theobald et al. 1997, Swenson and Franklin 2000).

Many recent studies focusing on the ecological impacts of nearshore residential development have examined inland lakes with less attention on Great Lakes shorelines (Rottenborn 1999, Lindsay et al. 2002, Elias and Meyer 2003). Perimeters of both inland lakes and the Great Lakes provide an interface between aquatic and

\footnotetext{
${ }^{1}$ School of Forest Resources and Environmental Science, Michigan Technological University, Houghton, MI 49931, USA.

${ }^{2}$ Current address: 73 Training Hill, Middletown, CT 06457, USA.

${ }^{3}$ Corresponding author; e-mail: michelle.ford@gza.com
}

upland ecosystems but, because of their size and associated climatological influence, the abiotic and biotic environment along Great Lakes shorelines often differs dramatically from inland lake riparian zones or adjoining upland habitats (Eichenlaub 1979, Albert et al. 1986). Thus, results from studies of inland lakes cannot be confidently extended to larger lake systems.

North America's Great Lakes (Huron, Ontario, Michigan, Erie, and Superior) contain 20\% of the world's and 95\% of North America's surface fresh water. The waters of the Great Lakes support the world's largest freshwater commercial fishery, supply drinking water to millions of citizens in the United States and Canada, and support a multimillion dollar recreation industry (Great Lakes Information Network 2005). The amenity values associated with the $17,542 \mathrm{~km}$ of Great Lakes shoreline have likewise attracted residential development (Schnaiberg et al. 2002). Shoreline residential development on the Great Lakes has accelerated in recent years and now represents one of the fastest growing segments of rural housing expansion in the region (Orr 1997).

The Keweenaw Peninsula is in the western Upper Peninsula of Michigan and is surrounded on three sides by Lake Superior (Fig. 1). Keweenaw County is one of the least populated in Michigan and much of the shoreline remains undeveloped; however, residential development in this area has rapidly increased over the past decade with population shifts to rural areas (Orr 1997). The Upper Peninsula has not yet felt the 
effects of rural in-migration as greatly as other scenic places in the United States, but land use changes and population increases are evident (Orr 1997).

We focused on differences between residentially developed and undeveloped shoreline areas of Lake Superior and measured vegetation habitat features associated with development known to influence bird presence and relative abundance. To our knowledge, this is the first study to examine the influence of Great Lakes shoreline residential development on forest breeding bird communities on any of the Great Lakes. Our objectives were to: (1) evaluate differences in forest vegetation between developed and undeveloped areas, (2) examine if relative abundance of breeding bird species differs between residentially developed and undeveloped shoreline forest along Lake Superior, and (3) assess the spatial scale at which development influences bird presence and abundance.

\section{METHODS}

Study Sites.-The study was conducted in two residentially developed and three undeveloped shoreline areas along the eastern shore of Lake Superior in the Keweenaw Peninsula in the northern-most part of the Michigan's Upper Peninsula (Fig. 1). Only east and southeast facing areas were used to minimize variability in soils, bedrock geology, site aspect, wind, and forest type. Developed areas included Hermit's Cove $\left(47^{\circ} 15^{\prime} \mathrm{N}, 88^{\circ} 07^{\prime} \mathrm{W}\right)$ and Rabbit Bay $\left(47^{\circ} 04^{\prime} \mathrm{N}\right.$, $88^{\circ} 20^{\prime} \mathrm{W}$ ) in Keweenaw and Houghton counties, respectively. These areas are similarly developed and are comprised predominately of seasonal cottages which are bounded on one side by Lake Superior and on the other by closed-canopy mixed hardwood and boreal transition forest with a gravel road along the forest side of the properties. Cottages at both sites are only on the lake side of the road and have a mixture of old (built prior to 1950) and newer homes, as well as seasonal and permanent residents. Most cottages are occupied primarily during the summer months (Manarolla 2005).

Low-elevation and near-shore portions of the Keweenaw Peninsula are dominated by boreal transition forests composed primarily of paper birch (Betula papyrifera), balsam fir (Abies balsamea), red maple (Acer rubrum), white spruce (Picea glauca), northern white cedar (Thuja occidentalis), and yellow birch (Betula allegha- niensis). Average precipitation for Keweenaw County during the breeding season from 1 May 2005 through 31 July 2005 was $\sim 22.8 \mathrm{~cm}$ with a mean temperature of $14^{\circ} \mathrm{C}$ (Weather Underground Corporation 2005). The soils of the eastern shore of the Keweenaw Peninsula range from gravelly and rocky sands to sandy loams and are poorly drained in lower elevations near the shore (Albert et al. 1986).

Undeveloped study areas, Smith Fisheries $\left(47^{\circ}\right.$ $\left.23^{\prime} \mathrm{N}, 87^{\circ} 53^{\prime} \mathrm{W}\right)$, south Rabbit Bay $\left(47^{\circ} 02^{\prime} \mathrm{N}\right.$, $\left.88^{\circ} 21^{\prime} \mathrm{W}\right)$, and north Hermit's Cove $\left(47^{\circ} 15^{\prime} \mathrm{N}\right.$, $88^{\circ} 06^{\prime} \mathrm{W}$ ) were selected by visiting areas along the eastern shore in early spring and by using Geographic Information System (GIS) data and aerial photographs to identify areas with and without concentrated residential development near the shore. Undeveloped study areas were along sections of shoreline north of Hermit's Cove and along sections of shoreline south of Rabbit Bay, and were placed $\geq 300 \mathrm{~m}$ from the nearest residential structure. The Smith Fisheries study area was $\sim 48 \mathrm{~km}$ north of Hermit's Cove, just north of the town of Lac La Belle, and was only accessible via logging roads and trails (Fig. 1). Thirty-six plots were established for vegetation and bird community sampling, 15 in undeveloped study areas and 21 in developed study areas. We collected data at two spatial scales: (1) broad-scale: developed and undeveloped shoreline study areas, and (2) fine-scale: developed and undeveloped sides of the road accessing developed shoreline study areas.

Measurement of Habitat Variables.-We measured habitat characteristics for the broad scale comparison along transects spaced every $100 \mathrm{~m}$ in both developed and undeveloped shoreline study areas. Vegetation transect centers along undeveloped shoreline study areas were $50 \mathrm{~m}$ inland. Transects along developed shoreline study areas were centered on the center of the unpaved road (50-75 $\mathrm{m}$ from the shore). We considered roads to be part of the vegetation disturbance associated with development. A second set of transects was oriented perpendicular to the shore, and extended $50 \mathrm{~m}$ in each direction toward and away from the shoreline for both developed and undeveloped shoreline study areas. This method sampled vegetation in both developed residential property and the undeveloped forest across the road from the development along the developed shoreline. Point-count centers for bird surveys were also placed in the center of the road so that equal 


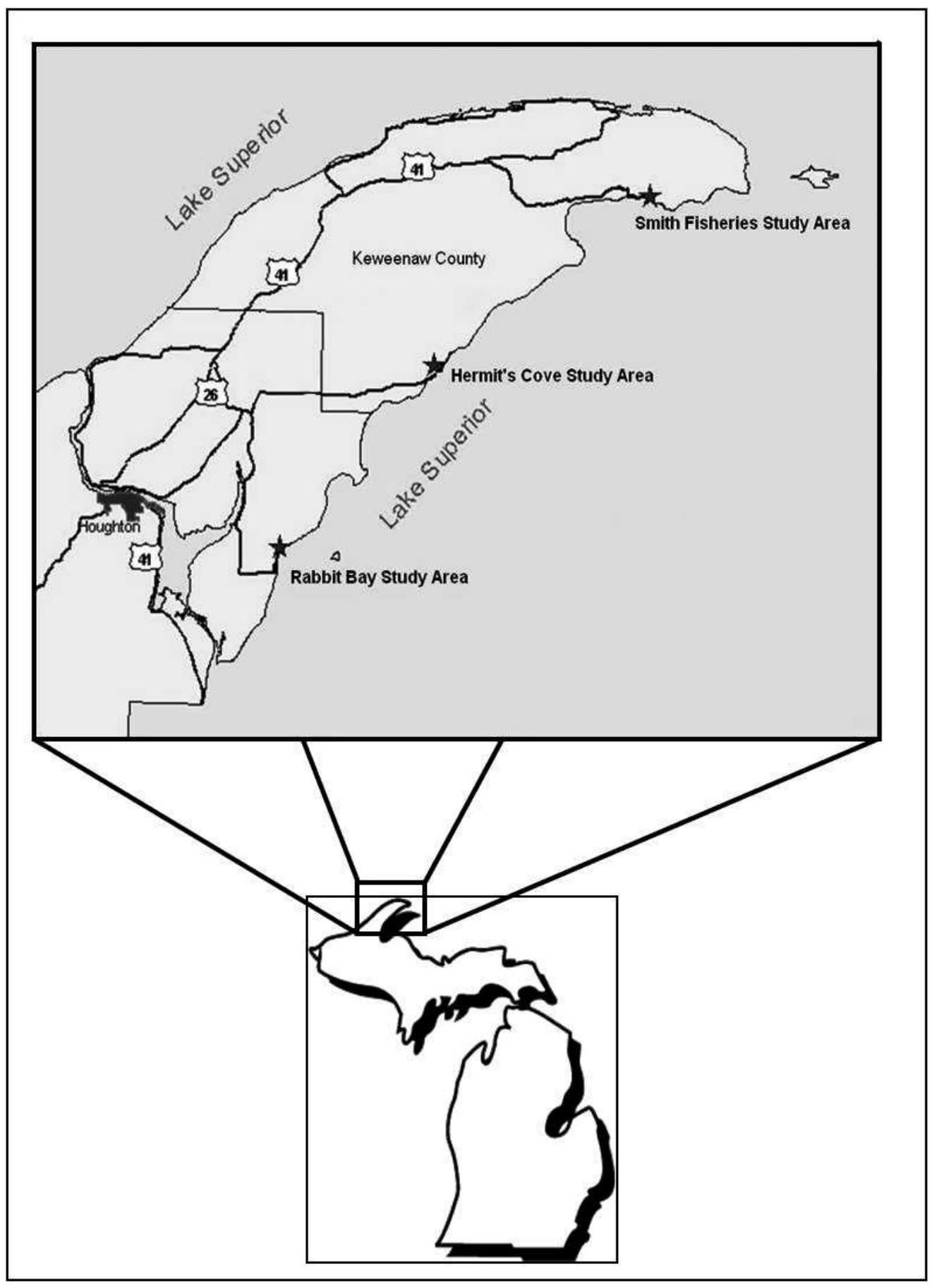

FIG. 1. Study sites in Houghton and Keweenaw counties, Michigan, USA. 
portions of the point-count area were in developed/shoreline and undeveloped/inland sides of the road.

Vegetation sampling methods were adapted from Noon (1981). Canopy cover and ground cover were estimated using an ocular tube approximately every $2 \mathrm{~m}$ along each transect. Coarse woody debris (CWD) was defined as any piece of downed wood $>8 \mathrm{~cm}$ in diameter that crossed a transect. The total pieces of CWD that crossed a transect were recorded and divided by the length of the transect $(100 \mathrm{~m})$. Diameter at breast height $(\mathrm{DBH})$ and species were recorded for all living trees $>3 \mathrm{~cm}$ in diameter within arms length, $\sim 0.8 \mathrm{~m}$, on either side of the transect. DBH data were converted to total stem count to compare the number of stems $>3 \mathrm{~cm}$ in each transect. We also calculated deciduous and coniferous shrub/sapling density along each transect in developed and undeveloped areas. Each woody stem $\leq 3 \mathrm{~cm}$ in diameter at breast height was recorded as either coniferous or deciduous. Average density for each shrub type was calculated as the total number of shrubs counted divided by the length of the transect. Total shrub density was calculated as the sum of both deciduous and coniferous shrubs divided by transect length.

A gridded density board was used to measure understory density and calculate percent cover from 0 to $3 \mathrm{~m}$ above the forest floor (Noon 1981). Separate density readings were taken facing areas on each side of the road (i.e., facing the residential development along the shoreline and facing the undeveloped inland) by standing on the side of the road with the density board placed $11.3 \mathrm{~m}$ from the road edge. Readings were summed and converted to percent cover in four height categories, $0-0.3,>0.3-1,>1-2$, and $>2 \mathrm{~m}$, and total understory density $0-3 \mathrm{~m}$.

Bird Surveys.-We used $5 \mathrm{~min}$ 50-m fixedradius point counts to estimate breeding bird relative abundance (Bibby et al. 2000, Sutherland et al. 2004) at developed and undeveloped sites. Counts started at sunrise, $\sim 0600 \mathrm{hrs}$, and ended by $1030 \mathrm{hrs}$ between 6 June and 1 July 2005 . Point-count centers were established every $200 \mathrm{~m}$ to minimize double counting individuals (Bibby et al. 2000). Point-count centers in developed shoreline study areas were in the center of the road at the center of vegetation transects, 50-75 m from the shoreline. Point-count centers within undeveloped shoreline study areas were $50 \mathrm{~m}$ from the shore to minimize wave noise interference. Twenty-one point-count stations were established in developed shoreline study areas and 15 in undeveloped shoreline study areas; all counts were done by M. T. Ford.

The observer waited for 2 min prior to start of each point-count period (Reynolds et al. 1980). All birds detected by sight or sound within the 50$\mathrm{m}$ radius within $5 \mathrm{~min}$ were recorded. Birds detected outside the 50-m radius or seen flying over were also noted, but were not included in analyses. All stations were visited two times in 2005 , once early and once later in the breeding season with one of the counts conducted within $30 \mathrm{~min}$ of sunrise and the other later in the morning. Point counts were not conducted in rain or high winds ( $>20 \mathrm{~km} / \mathrm{hr}$ ) (Robbins 1981).

Bird species richness in developed and undeveloped areas was estimated from the total number of species detected in 2005. Relative abundance for each species was defined as the maximum number of individuals detected between the two visits at each station. Birds were also grouped into nesting/foraging guilds to investigate guild-level associations with development.

Statistical Analysis.-We used $t$ - and Wilcoxon-Mann-Whitney non-parametric tests to examine differences in bird abundance and vegetation attributes between developed and undeveloped areas. The more powerful $t$-test was used when substantial deviation from symmetry was not detected in boxplots generated from the raw data (Quinn and Keough 2002). The Wilcoxon-Mann-Whitney test was used at the same significance levels to examine nesting/ foraging guild differences between developed and undeveloped areas. Understory density from 0 to $0.3 \mathrm{~m}$ and 1 to $2 \mathrm{~m}$ appeared to be asymmetrical and was analyzed using WilcoxonMann-Whitney tests. The Shannon-Wiener species diversity index $\left(H^{\prime}\right)$ was used to compare species richness and evenness between developed and undeveloped areas (Magurran 2004). All data were tested at a significance level of $\alpha=0.05$ unless otherwise specified.

\section{RESULTS}

Broad Spacial Scale Comparison of Habitat Characteristics in Developed and Undeveloped Shoreline Study Areas.-Developed shoreline had higher deciduous and total shrub density than undeveloped areas. Canopy cover and CWD were 
TABLE 1. Vegetation characteristics in developed and undeveloped shoreline study areas on the Keweenaw Peninsula, Michigan.

\begin{tabular}{|c|c|c|c|}
\hline Characteristics & Treatment & Mean \pm SD & $P$ \\
\hline Canopy cover ${ }^{\mathrm{a}}$ & $\begin{array}{l}\text { Developed } \\
\text { Undeveloped }\end{array}$ & $\begin{array}{l}76.91 \pm 22.33 \\
87.82 \pm 12.08\end{array}$ & $<0.000$ \\
\hline Ground cover & $\begin{array}{l}\text { Developed } \\
\text { Undeveloped }\end{array}$ & $\begin{array}{l}64.68 \pm 16.42 \\
61.56 \pm 19.16\end{array}$ & 0.312 \\
\hline Coarse woody debris ${ }^{\mathrm{a}}$ & $\begin{array}{l}\text { Developed } \\
\text { Undeveloped }\end{array}$ & $\begin{array}{l}8.38 \pm 5.75 \\
16.6 \pm 10.16\end{array}$ & $<0.000$ \\
\hline Number of stems $>8 \mathrm{~cm}^{\mathrm{a}}$ & $\begin{array}{l}\text { Developed } \\
\text { Undeveloped }\end{array}$ & $\begin{array}{l}357 \pm 205.96 \\
288 \pm 166.13\end{array}$ & $<0.000$ \\
\hline Total shrub density ${ }^{\mathrm{a}}$ & $\begin{array}{l}\text { Developed } \\
\text { Undeveloped }\end{array}$ & $\begin{aligned} 6.3 & \pm 10.62 \\
4.38 & \pm 9.24\end{aligned}$ & 0.016 \\
\hline Deciduous shrub density ${ }^{\mathrm{a}}$ & $\begin{array}{l}\text { Developed } \\
\text { Undeveloped }\end{array}$ & $\begin{array}{l}9.38 \pm 8.25 \\
4.35 \pm 6.18\end{array}$ & $<0.000$ \\
\hline Coniferous shrub density & $\begin{array}{l}\text { Developed } \\
\text { Undeveloped }\end{array}$ & $\begin{array}{l}3.23 \pm 4.16 \\
4.42 \pm 6.05\end{array}$ & 0.194 \\
\hline Total understory density (UD) & $\begin{array}{l}\text { Developed } \\
\text { Undeveloped }\end{array}$ & $\begin{array}{r}50.03 \pm 28.25 \\
50.9 \pm 33.21\end{array}$ & 0.87 \\
\hline UD $0-0.3 \mathrm{~m}$ & $\begin{array}{l}\text { Developed } \\
\text { Undeveloped }\end{array}$ & $\begin{array}{r}78.19 \pm 36.31 \\
74 \pm 34.17\end{array}$ & 0.483 \\
\hline $\mathrm{UD}>0.3-1.0 \mathrm{~m}$ & $\begin{array}{l}\text { Developed } \\
\text { Undeveloped }\end{array}$ & $\begin{array}{l}51.85 \pm 37.2 \\
47.04 \pm 40.85\end{array}$ & 0.473 \\
\hline $\mathrm{UD}>1.0-2.0 \mathrm{~m}$ & $\begin{array}{l}\text { Developed } \\
\text { Undeveloped }\end{array}$ & $\begin{array}{l}27.83 \pm 34.07 \\
35.63 \pm 37.13\end{array}$ & 0.203 \\
\hline $\mathrm{UD}>2.0-3.0 \mathrm{~m}$ & $\begin{array}{l}\text { Developed } \\
\text { Undeveloped }\end{array}$ & $\begin{array}{l}42.24 \pm 36.7 \\
46.93 \pm 37.88\end{array}$ & 0.462 \\
\hline Total diameter at breast height $(\mathrm{DBH})$ & $\begin{array}{l}\text { Developed } \\
\text { Undeveloped }\end{array}$ & $\begin{array}{l}17.77 \pm 15.87 \\
19.51 \pm 59.66\end{array}$ & 0.498 \\
\hline $\mathrm{DBH}$ of deciduous trees & $\begin{array}{l}\text { Developed } \\
\text { Undeveloped }\end{array}$ & $\begin{array}{l}16.65 \pm 15.18 \\
21.41 \pm 96.54\end{array}$ & 0.474 \\
\hline $\mathrm{DBH}$ of coniferous trees & $\begin{array}{l}\text { Developed } \\
\text { Undeveloped }\end{array}$ & $\begin{array}{r}18.92 \pm 16.47 \\
39.4 \pm 13.52\end{array}$ & 0.635 \\
\hline
\end{tabular}

\footnotetext{
${ }^{\text {a }}$ Significant $(\alpha=0.05)$.
}

greater in undeveloped than developed areas. Developed and undeveloped shoreline study areas did not differ in mean DBH for all trees together, deciduous trees, or coniferous trees. Stem counts were greater in developed than undeveloped shoreline study areas (Table 1).

Small Spatial Scale Comparison of Developed Shoreline and Undeveloped Inland Sides of Shoreline Road.-The residentially developed shoreline side of the road had lower canopy cover, ground cover, CWD, stem counts, total understory density, and understory density from $0-0.3$ to $>0.3-1 \mathrm{~m}$ (Table 2). Mean DBH of coniferous trees, deciduous trees, and all trees pooled did not differ between developed and undeveloped sides of the road (Table 2).

Birds.-We detected 291 individuals of 32 species for all point counts pooled within the $50-\mathrm{m}$ radius point counts during the survey period (Table 3). Species richness was $\sim 34 \%$ greater in developed (29 species) shoreline study areas than undeveloped (18 species) shoreline study areas. Ninety percent ( 29 of 32 species) of all species detected were in developed shoreline study areas; only 56\% (18 of 32 species) of all species detected were in undeveloped shoreline study areas. Fourteen species were found only in developed shoreline study areas and three species were only in undeveloped shoreline study areas (Table 3). The Shannon-Wiener diversity index $\left(H^{\prime}\right)$ was 2.96 in developed and 2.45 in undeveloped shoreline study areas. Species evenness was virtually identical in developed $(E=0.88)$ and undeveloped $(E=0.86)$ shoreline study areas.

The Shannon-Wiener diversity index within developed shoreline study areas was 2.97 on the developed side of the road and 2.70 on the inland, undeveloped side of the road. Species evenness was similar between shoreline $(E=0.92)$ and inland $(E=0.87)$ sides of the road. 
TABLE 2. Vegetation characteristics in developed shoreline and undeveloped inland sides of shoreline access roads in residentially developed areas on the Keweenaw Peninsula, Michigan.

\begin{tabular}{|c|c|c|c|}
\hline Characteristics & Treatment & Mean \pm SD & $P$ \\
\hline Canopy cover ${ }^{\mathrm{a}}$ & $\begin{array}{l}\text { Shoreline } \\
\text { Inland }\end{array}$ & $\begin{array}{l}69.49 \pm 23.31 \\
84.34 \pm 18.79\end{array}$ & 0.002 \\
\hline Ground cover ${ }^{\mathrm{a}}$ & $\begin{array}{l}\text { Shoreline } \\
\text { Inland }\end{array}$ & $\begin{array}{l}68.16 \pm 18.9 \\
69.49 \pm 12.81\end{array}$ & 0.005 \\
\hline Coarse woody debris ${ }^{\mathrm{a}}$ & $\begin{array}{l}\text { Shoreline } \\
\text { Inland }\end{array}$ & $\begin{array}{r}6.71 \pm 6.16 \\
10.05 \pm 4.83\end{array}$ & 0.008 \\
\hline Number of stems $>8 \mathrm{~cm}^{\mathrm{a}}$ & $\begin{array}{l}\text { Shoreline } \\
\text { Inland }\end{array}$ & $\begin{array}{r}138 \pm 79.53 \\
219.5 \pm 126.5\end{array}$ & $<0.001$ \\
\hline Total shrub density & $\begin{array}{l}\text { Shoreline } \\
\text { Inland }\end{array}$ & $\begin{array}{r}5.51 \pm 6.99 \\
7.1 \pm 6.66\end{array}$ & 0.16 \\
\hline Deciduous shrub density & $\begin{array}{l}\text { Shoreline } \\
\text { Inland }\end{array}$ & $\begin{array}{r}7.71 \pm 8.07 \\
11.05 \pm 8.19\end{array}$ & 0.066 \\
\hline Coniferous shrub density & $\begin{array}{l}\text { Shoreline } \\
\text { Inland }\end{array}$ & $\begin{array}{l}3.32 \pm 4.79 \\
3.15 \pm 3.48\end{array}$ & 0.854 \\
\hline Total understory density ${ }^{a}$ & $\begin{array}{l}\text { Shoreline } \\
\text { Inland }\end{array}$ & $\begin{array}{r}43.86 \pm 39.86 \\
56.2 \pm 40.33\end{array}$ & 0.047 \\
\hline UD $0-0.3 \mathrm{~m}^{\mathrm{a}}$ & $\begin{array}{l}\text { Shoreline } \\
\text { Inland }\end{array}$ & $\begin{aligned} 67.88 & \pm 40.81 \\
88.5 & \pm 28.06\end{aligned}$ & 0.01 \\
\hline $\mathrm{UD}>0.3-1.0 \mathrm{~m}^{\mathrm{a}}$ & $\begin{array}{l}\text { Shoreline } \\
\text { Inland }\end{array}$ & $\begin{array}{l}41.11 \pm 35.9 \\
62.59 \pm 35.73\end{array}$ & 0.008 \\
\hline $\mathrm{UD}>1.0-2.0 \mathrm{~m}$ & $\begin{array}{l}\text { Shoreline } \\
\text { Inland }\end{array}$ & $\begin{array}{l}25.32 \pm 32.19 \\
30.34 \pm 36.08\end{array}$ & 0.508 \\
\hline $\mathrm{UD}>2.0-3.0 \mathrm{~m}$ & $\begin{array}{l}\text { Shoreline } \\
\text { Inland }\end{array}$ & $\begin{array}{l}41.12 \pm 35.07 \\
43.37 \pm 38.67\end{array}$ & 0.784 \\
\hline Total diameter at breast height $(\mathrm{DBH})$ & $\begin{array}{l}\text { Shoreline } \\
\text { Inland }\end{array}$ & $\begin{array}{l}18.75 \pm 16.21 \\
17.15 \pm 15.64\end{array}$ & 0.194 \\
\hline DBH of deciduous trees & $\begin{array}{l}\text { Shoreline } \\
\text { Inland }\end{array}$ & $\begin{array}{l}18.13 \pm 14.13 \\
15.97 \pm 15.63\end{array}$ & 0.21 \\
\hline DBH of coniferous trees & $\begin{array}{l}\text { Shoreline } \\
\text { Inland }\end{array}$ & $\begin{array}{r}19.2 \pm 17.56 \\
18.68 \pm 15.55\end{array}$ & 0.77 \\
\hline
\end{tabular}

${ }^{\mathrm{a}}$ Significant $(\alpha=0.05)$.

Broad Spatial Scale Comparison of Bird Species Relative Abundance.-Only Red-eyed Vireos (Vireo olivaceus), of 32 total species detected, were significantly (5 times) more abundant in developed than undeveloped shoreline study areas. Ground-nesting birds were equally abundant in developed and undeveloped shoreline study areas $(P>0.05)$. Shrub nesters were more abundant in undeveloped forest and canopy nesters were more abundant in developed shoreline study areas when species were pooled based on nest site location $(P<0.05)$ (Table 3$)$.

Fine-scale Comparison of Bird Species Relative Abundance.-Two species, American Crow (Corvus brachyrhynchos) and American Robin (Turdus migratorius) were statistically more abundant on the developed, shoreline side of the shoreline access roads. Three species, Black-throated Green Warbler (Dendroica virens), Red-eyed Vireo, and
Red-breasted Nuthatch (Sitta canadensis) were more abundant on the undeveloped, inland side of shoreline access roads (Table 4). Differences in relative abundance of American Redstarts (Setophaga ruticilla) and Blackburnian Warblers (Dendroica fusca $)$ approached significance $(P=0.10)$ with both detected more frequently on the undeveloped, inland side of point counts (Table 4).

\section{DISCUSSION}

Analyses of developed and undeveloped shoreline areas at the broad scale showed that only one species was significantly more abundant in developed shoreline areas and no species were significantly more abundant in undeveloped shoreline areas. Fine-scale plot-level analyses in developed areas suggested that two species were significantly more abundant on the developed, 
TABLE 3. Bird species detected on point counts between May and July 2005 in developed and undeveloped study areas on the Keweenaw Peninsula, Michigan.

\begin{tabular}{|c|c|c|c|c|c|c|c|}
\hline \multirow[b]{2}{*}{ Common name } & \multirow[b]{2}{*}{ Species } & \multirow[b]{2}{*}{ AOU Code } & \multicolumn{2}{|c|}{ Number of individuals detected } & \multicolumn{3}{|c|}{ Nesting guild } \\
\hline & & & Developed & Undeveloped & Ground & Shrub & Canopy \\
\hline American Crow & $\begin{array}{l}\text { Corvus } \\
\quad \text { brachyrhynchos }\end{array}$ & AMCR & 5 & 0 & & & $\mathrm{x}$ \\
\hline American Goldfinch & Spinus tristis & AMGO & 1 & 0 & & $\mathrm{x}$ & \\
\hline American Redstart & Setophaga ruticilla & AMRE & 14 & 10 & & & $\mathrm{x}$ \\
\hline American Robin & Turdus migratorius & AMRO & 9 & 0 & & & $\mathrm{x}$ \\
\hline Black-and-white Warbler & Mniotilta varia & BAWW & 7 & 3 & $\mathrm{x}$ & & \\
\hline Black-capped Chickadee & Poecile atricapillus & $\mathrm{BCCH}$ & 6 & 0 & & & $\mathrm{x}$ \\
\hline Blackburnian Warbler & Dendroica fusca & BLBW & 1 & 0 & & & $\mathrm{x}$ \\
\hline Blue Jay & Cyanocitta cristata & BLJA & 2 & 1 & & & $\mathrm{x}$ \\
\hline Black-throated Green Warbler & Dendroica virens & BTNW & 7 & 3 & & & $\mathrm{x}$ \\
\hline Chipping Sparrow & Spizella passerina & CHSP & 3 & 0 & & $\mathrm{x}$ & \\
\hline Cape May Warbler & Dendroica tigrina & CMWA & 0 & 1 & & & $\mathrm{x}$ \\
\hline Common Raven & Corvus corax & CORA & 0 & 2 & & & $\mathrm{x}$ \\
\hline Chestnut-sided Warbler & $\begin{array}{l}\text { Dendroica } \\
\quad \text { pensylvanica }\end{array}$ & CSWA & 1 & 0 & & $\mathrm{x}$ & \\
\hline Golden-crowned Kinglet & Regulus satrapa & GCKI & 6 & 1 & & & $\mathrm{x}$ \\
\hline Hairy Woodpecker & Picoides villosus & HAWO & 2 & 0 & & & $\mathrm{x}$ \\
\hline Hermit Thrush & Catharus guttatus & HETH & 3 & 10 & $\mathrm{x}$ & & \\
\hline Mallard & Anas platyrhynchos & MALL & 2 & 0 & $\mathrm{x}$ & & \\
\hline Magnolia Warbler & Dendroica magnolia & MAWA & 1 & 1 & & & $\mathrm{x}$ \\
\hline Yellow-rumped Warbler & D. coronata & MYWA & 18 & 4 & & & $\mathrm{x}$ \\
\hline Nashville Warbler & Vermivora ruficapilla & NAWA & 23 & 24 & $\mathrm{x}$ & & \\
\hline Northern Parula & Parula americana & NOPA & 24 & 12 & & & $\mathrm{x}$ \\
\hline Ovenbird & Seiurus aurocapilla & OVEN & 9 & 0 & $\mathrm{x}$ & & \\
\hline Pine Warbler & Dendroica pinus & PIWA & 3 & 0 & & & $\mathrm{x}$ \\
\hline Red-breasted Nuthatch & Sitta canadensis & RBNU & 5 & 3 & & & $\mathrm{x}$ \\
\hline Ruby-crowned Kinglet & Regulus calendula & RCKI & 0 & 1 & & & $\mathrm{x}$ \\
\hline Red-eyed Vireo & Vireo olivaceus & REVI & 14 & 3 & & & $\mathrm{x}$ \\
\hline Ruby-throated Hummingbird & Archilochus colubris & RTHU & 2 & 0 & & & $\mathrm{x}$ \\
\hline Song Sparrow & Melospiza melodia & SOSP & 1 & 0 & $\mathrm{x}$ & & \\
\hline Swainson's Thrush & Catharus ustulatus & SWTH & 3 & 8 & & & $\mathrm{x}$ \\
\hline White-throated Sparrow & Zonotrichia albicollis & WTSP & 7 & 0 & $\mathrm{x}$ & & \\
\hline White-breasted Nuthatch & Sitta carolinensis & WBNU & 1 & 0 & & & $\mathrm{x}$ \\
\hline Winter Wren & $\begin{array}{l}\text { Troglodytes } \\
\text { troglodytes }\end{array}$ & WIWR & 6 & 13 & & $\mathrm{x}$ & \\
\hline
\end{tabular}

shoreline portion rather than the undeveloped, inland portion of the shoreline access roads. Three species were more abundant on the inland, undeveloped side of the shoreline access roads. Our results suggest species may be responding to habitat alterations associated with residential development at the patch, rather than the larger stand/landscape scale. We found developmentrelated differences in natural habitat features including canopy volume, shrub cover, forest floor vegetative cover, and amount of CWD.

Riparian areas in much of the U.S. represent concentrations of biodiversity and foci for residential development in rural areas (Naiman et al. 1993, Schnaiberg et al. 2002, Bub et al. 2004,
Gonzalez-Abraham et al. 2006). Shoreline vegetation, compared to adjoining inland sites, is typically denser, more varied in species composition, and offers greater vertical and horizontal structural diversity (Riffell et al. 2001). Rottenborn (1999) proposed that shoreline forests have unique vegetation characteristics compared to similar forests distant from the shoreline and these differences may shape patterns of bird community composition. Research has shown that riparian vegetation structure influences breeding bird communities (Willson 1974, Hostetler and Holling 2000) and altering these habitats through removal of native vegetation can change bird community structure. Vegetation structure fre- 
TABLE 4. Birds detected on undeveloped inland and developed shoreline sides of shoreline access roads in residentially developed areas on the Keweenaw Peninsula, Michigan.

\begin{tabular}{|c|c|c|c|c|}
\hline Species & Treatment & $\begin{array}{l}\text { Total } \\
\text { count }\end{array}$ & Mean/10 plots $\pm S D$ & Zcalc $^{\mathrm{a}}$ \\
\hline \multirow[t]{2}{*}{$\mathrm{AMCR}^{\mathrm{a}}$} & Inland & 0 & $0 \pm 0$ & 2.03 \\
\hline & Shoreline & 5 & $23.8 \pm 0.54$ & \\
\hline \multirow[t]{2}{*}{ AMGO } & Inland & 0 & $0 \pm 0$ & 0.97 \\
\hline & Shoreline & 1 & $4.8 \pm 0.22$ & \\
\hline \multirow[t]{2}{*}{ AMRE } & Inland & 17 & $33.3 \pm 0.66$ & 1.91 \\
\hline & Shoreline & 7 & $28.6 \pm 0.56$ & \\
\hline \multirow[t]{2}{*}{ AMRO } & Inland & 1 & $0 \pm 0$ & 1.97 \\
\hline & Shoreline & 8 & $38.1 \pm 0.74$ & \\
\hline \multirow[t]{2}{*}{$\mathrm{BCCH}$} & Inland & 1 & $4.8 \pm 0.22$ & 1.37 \\
\hline & Shoreline & 4 & $19 \pm 0.4$ & \\
\hline \multirow[t]{2}{*}{ BLBW } & Inland & 2 & $9.5 \pm 0.3$ & 1.74 \\
\hline & Shoreline & 0 & $0 \pm 0$ & \\
\hline \multirow[t]{2}{*}{ BLJA } & Inland & 1 & $4.8 \pm 0.22$ & 1.01 \\
\hline & Shoreline & 2 & $9.5 \pm 0.3$ & \\
\hline \multirow[t]{2}{*}{ BTNW $^{\mathrm{a}}$} & Inland & 6 & $28.6 \pm 0.56$ & 2.04 \\
\hline & Shoreline & 2 & $9.5 \pm 0.3$ & \\
\hline \multirow[t]{2}{*}{ BAWW } & Inland & 3 & $14.3 \pm 0.36$ & 0.04 \\
\hline & Shoreline & 5 & $23.8 \pm 0.44$ & \\
\hline \multirow[t]{2}{*}{ CHSP } & Inland & 1 & $4.8 \pm 0.22$ & 0.57 \\
\hline & Shoreline & 2 & $9.5 \pm 0.3$ & \\
\hline \multirow[t]{2}{*}{ CSWA } & Inland & 0 & $0 \pm 0$ & 0.97 \\
\hline & Shoreline & 1 & $4.8 \pm 0.22$ & \\
\hline \multirow[t]{2}{*}{ CORA } & Inland & 0 & $0 \pm 0$ & 1.4 \\
\hline & Shoreline & 0 & $5.6 \pm 0.23$ & \\
\hline \multirow[t]{2}{*}{ GCKI } & Inland & 4 & $19 \pm 0.4$ & 1.17 \\
\hline & Shoreline & 2 & $9.5 \pm 0.3$ & \\
\hline \multirow[t]{2}{*}{ HAWO } & Inland & 1 & $4.8 \pm 0.22$ & -0.02 \\
\hline & Shoreline & 1 & $4.8 \pm 0.22$ & \\
\hline \multirow[t]{2}{*}{ HETH } & Inland & 0 & $0 \pm 0$ & 0.2 \\
\hline & Shoreline & 2 & $9.5 \pm 0.3$ & \\
\hline \multirow[t]{2}{*}{ MALL } & Inland & 0 & $0 \pm 0$ & 0.97 \\
\hline & Shoreline & 2 & $9.5 \pm 0.44$ & \\
\hline \multirow[t]{2}{*}{ MAWA } & Inland & 1 & $4.8 \pm 0.22$ & 1.4 \\
\hline & Shoreline & 0 & $0 \pm 0$ & \\
\hline \multirow[t]{2}{*}{ NAWA $^{\mathrm{a}}$} & Inland & 14 & $70 \pm 0.66$ & 4.2 \\
\hline & Shoreline & 8 & $38.1 \pm 0.59$ & \\
\hline \multirow[t]{2}{*}{ NOPA $^{a}$} & Inland & 21 & $100 \pm 0.95$ & 4.02 \\
\hline & Shoreline & 4 & $19 \pm 0.4$ & \\
\hline \multirow[t]{2}{*}{ OVEN } & Inland & 6 & $28.6 \pm 0.64$ & 0.45 \\
\hline & Shoreline & 3 & $14.3 \pm 0.36$ & \\
\hline PIWA & Inland & 1 & $4.8 \pm 0.22$ & -0.02 \\
\hline & Shoreline & 1 & $4.8 \pm 0.22$ & \\
\hline $\mathrm{RBNU}^{\mathrm{a}}$ & Inland & 4 & $19 \pm 0.4$ & 2.22 \\
\hline & Shoreline & 1 & $4.8 \pm 0.22$ & \\
\hline $\mathrm{REVI}^{\mathrm{a}}$ & Inland & 10 & $47.6 \pm 0.51$ & 2.47 \\
\hline & Shoreline & 4 & $19 \pm 0.40$ & \\
\hline RCKI & Inland & 1 & $2.8 \pm 0.17$ & 0.97 \\
\hline & Shoreline & 0 & $0 \pm 0$ & \\
\hline RTHU & Inland & 2 & $9.5 \pm 0.30$ & 1.40 \\
\hline & Shoreline & 0 & $0 \pm 0$ & \\
\hline SOSP & Inland & 0 & $0 \pm 0$ & 1.4 \\
\hline & Shoreline & 2 & $9.5 \pm 0.3$ & \\
\hline
\end{tabular}

TABLE 4. Continued.

\begin{tabular}{ccccc}
\hline Species & Treatment & $\begin{array}{c}\text { Total } \\
\text { count }\end{array}$ & Mean/10 plots \pm SD & Zcalc $^{\mathrm{a}}$ \\
\hline SWTH & Inland & 2 & $9.5 \pm 0.3$ & 0.7 \\
& Shoreline & 1 & $4.8 \pm 0.22$ & \\
WBNU & Inland & 1 & $4.8 \pm 0.22$ & 0.97 \\
& Shoreline & 0 & $0 \pm 0$ & \\
WIWR $^{\mathrm{a}}$ & Inland & 5 & $24 \pm 0.44$ & 3.58 \\
& Shoreline & 1 & $5 \pm 0.22$ & \\
WTSP & Inland & 3 & $14 \pm 0.36$ & 0.73 \\
& Shoreline & 5 & $24 \pm 0.44$ & \\
MYWA & Inland & 10 & $48 \pm 0.68$ & 0.71 \\
& Shoreline & 9 & $43 \pm 0.51$ & \\
\hline
\end{tabular}

${ }^{\mathrm{a}}$ Mann Whitney Zcrit $=1.96$ at $\alpha=0.05$ and Zcrit $=1.645$ at $\alpha=0.10$. Ho is rejected when Zcalc $>$ Zcrit.

quently influences bird species richness, relative abundance (Willson 1974, Hostetler and Holling 2000), and foraging behavior (Smith et al. 1998). We found development-related differences in natural habitat features including canopy volume, shrub cover, forest floor vegetative cover, and amount of CWD. Research has shown that natural habitat features such as canopy volume, shrub cover, and forest vegetative cover are influenced by residential development (Clark et al. 1984, Christensen et al. 1996, Woodford and Meyer 2003). Many studies (Willson 1974, Burke and Nol 1998, Sallabanks et al. 2000) have suggested that changes in the avian community in the presence of human development are the result of development-related changes in vegetation rather than direct human disturbance of birds.

Elias and Meyer (2003) found that undeveloped shoreline in northern Wisconsin had higher percent canopy, subcanopy, understory cover, coarse woody debris, and percentage of shoreline overhung by trees and shrubs compared to developed shoreline stretches. They also found that plant species richness and diversity were greater in developed than in undeveloped shoreline areas. Lindsay et al. (2002) compared bird community attributes in riparian areas along developed and undeveloped inland lakes in northern Wisconsin. They reported that granivorous and omnivorous bird species were more abundant along developed lakes than undeveloped lakes. In contrast, insectivorous species were more abundant along undeveloped than developed lakeshores (Lindsay et al. 2002). Rottenborn (1999) reported that shoreline development near riparian areas in California affected nearby riparian bird communities and concluded that 
species richness and diversity decreased as native vegetation was lost to residential development.

Differences in vegetation characteristics between developed and undeveloped shoreline areas may be responsible for most of the differences we observed in bird abundance. Deciduous shrub density and total shrub density were greater along developed shoreline, while mean canopy cover and mean number of CWD pieces were greater along undeveloped shoreline. The greater number of all but coniferous shrubs and saplings within developed shoreline areas is likely the result of greater light penetration from the more open forest canopy. Comparison of forest characteristics at the individual plot scale suggested that within-plot canopy cover, CWD, understory density from 0 to $0.3 \mathrm{~m},>0.3$ to $1 \mathrm{~m}$, and overall understory density were greater on the undeveloped side of shoreline access roads. The presence of development features such as lawns, houses, outbuildings, walkways and driveways may contribute to these differences.

Christiansen et al. (1996) found that inland lake shoreline areas in northern Wisconsin with more cabins had fewer large pieces of dead and down wood in near shore waters, and speculated that many property owners remove snags and downed wood in or near the water's edge. The same process may occur around cottages, decreasing the amount of CWD in these areas. All study areas have thin soils which, given the close proximity to winds off Lake Superior, may result in increased blow-downs in developed shoreline areas, heightening the contrast in CWD abundance between developed and undeveloped shorelines.

The higher abundance of CWD, canopy cover, understory density from 0 to $0.3 \mathrm{~m}$ and from $>0.3$ to $1.0 \mathrm{~m}$ available in the undeveloped inland side of shoreline access road census locations likely influenced the relative abundance of several species. Nashville Warblers (Vermivora ruficapilla) and Winter Wrens (Troglodytes troglodytes) were both more abundant in the undeveloped inland portion of shoreline access roads. Nashville Warblers nest at the ground level and Winter Wrens often place their nests in the roots of upturned trees or snags (Ehrlich et. al. 1988). The higher abundance of CWD and increased understory density on the undeveloped, inland side of the road may be important habitat features for these two species.

Northern Parula (Parula americana) have been shown to prefer riparian areas (Moldenhauer and
Regelski 1996) and to be relatively tolerant of habitat disturbance short of clear cutting (Brooks 1940). Northern Parula, which build nests in the mid-canopy, have been shown to be positively correlated with at least $75 \%$ canopy cover and, when breeding, are heavily dependent on an abundance of epiphytes, particularly lichen (Usnea spp.) in their northern range (Collins et al. 1982, Moldenhauer and Regelski 1996). Northern Parula were more abundant on the inland, undeveloped side of shoreline access roads than on the residentially developed side. This may be the result of lower abundance of epiphytic lichen related to canopy openings (Esseen and Rehhorn 1998) and/or preference by Northern Parula for increased canopy cover, which was greater on the inland side of point-count plots.

Red-breasted Nuthatches depend on the availability of cavities in dead standing trees or roots of upturned trees as well as sap from living conifers. The sap is used to coat the entrance of the cavity during the incubation period to protect against nest predators (Ghalambor and Martin 1999). The increased abundance of CWD in inland areas may be an indicator of dead, dying trees in the vicinity and perhaps of standing dead, cavity bearing trees making it an increasingly suitable habitat for Redbreasted Nuthatches.

Given that our data were collected over a single year, we realize there are several limitations associated with our conclusions. Annual variations in weather, vegetation characteristics associated with development (i.e., possible collection of CWD by landowners for firewood or annual maintenance of vegetation within residential properties), and local bird community all likely contribute to bird species response within our study areas. Another recognized limitation is the potential for birds to naturally nest $>50 \mathrm{~m}$ from the shoreline, regardless of the presence of residential development. Species detection is another factor which may be identified as a limitation of our study; it is likely that differences exist in detection of bird species between the more open, less vegetated residential shoreline areas and undeveloped areas with overall greater sub-canopy density. We believe the differences found in bird species abundance between residentially developed and undeveloped shoreline areas are legitimate based upon the known species habitat preferences and the measured vegetation characteristics of each study area. 
It is essential to clarify the scale at which bird species respond to changes related to shoreline development to understand how developmentrelated habitat alteration interacts with breeding bird habitat use. Our results suggest birds may respond more to development-related habitat changes at the scale of tens of meters, than at the scale of the shoreline study areas we measured (i.e., hundreds of meters).

Ornithologists and ecologists have long recognized the role that scale has in understanding habitat use in wildlife (Wiens 1989, Levin 1992). The differing patterns of species composition and relative abundance we found suggest development related changes did not elicit a strong response by most bird species at the scale of the $1 \mathrm{~km}$ shoreline. However, development related changes likely influence which areas (e.g., developed or undeveloped side of the road) within a shoreline area a bird chooses for breeding. Our study was conducted in a landscape matrix that is $>95 \%$ forested, and any forest opening related to residential development represents a relatively small landscape disturbance. Landscape condition has also been shown to be a strong predictor of bird species presence (Saab 1999, Lee et al. 2002). Our results should be viewed in the context of the largely unfragmented surrounding forest.

\section{ACKNOWLEDGMENTS}

This research was supported by the USDA McIntireStennis Program and the Copper Country Audubon Society. Robert Froese, Alec Lindsay, Hugh Gorman, and Tom Drummer were helpful in revising earlier drafts of this manuscript. Kurt Doran and Kevin Ford assisted with vegetation data collection.

\section{LITERATURE CITED}

Albert, D. A., S. R. Denton, And B. V. Barnes. 1986. Regional landscape ecosystems of Michigan. School of Natural Resources, University of Michigan, Ann Arbor, USA.

Bibby, C. J., N. D. Burgess, D. A. Hill, And S. H. Mustoe. 2000. Bird census techniques. Academic Press, London, United Kingdom.

BRoOKS, M. 1940. The breeding warblers of the central Allegheny Mountain Region. Wilson Bulletin 52:249266.

BRown, D. G. 2003. Land use and forest cover on private parcels in the upper Midwest USA, 1970 to 1990. Landscape Ecology 18:777-790.

Bub, B. R., D. J. Flaspohler, and C. J. Huckins. 2004 Riparian and upland breeding-bird assemblages along headwater streams in Michigan's Upper Peninsula. Journal of Wildlife Management 68:383-392.
Burke, D. A. AND E. Nol. 1998. Influence of food abundance, nest-site habitat, and forest fragmentation on breeding Ovenbirds. Auk 115:96-104.

Christensen, D. L., B. R. Herwig, D. E. Schindler, And S. R. CARPENTER. 1996. Impacts of lakeshore residential development on coarse woody debris in north temperate lakes. Ecological Applications 6:1143-1149.

Clark, K. L., D. L. Euler, And E. Armstrong. 1984. Predicting avian community response to lakeshore cottage development. Journal of Wildlife Management 48:1239-1247.

Collins, S. L., F. C. JAMES, AND P. G. Risser. 1982. Habitat relationships of wood warblers (Parulidae) in northern central Minnesota. Oikos 39:50-58.

Ehrlich, P. R., D. S. Dobkin, AND D. Wheye. 1988. The birders handbook: a field guide to the natural history of North American birds. Simon and Schuster Inc., New York, USA.

EICHENlauB, V. 1979. Weather and climate of the Great Lakes Region. Notre Dame Press, South Bend, Indiana, USA.

Elias, J. E. AND M. W. Meyer. 2003. Comparisons of undeveloped and developed shorelands, northern Wisconsin, and recommendations for restoration. Wetlands 23:800-816.

EsseEn, P. AND K. RENHORN. 1998. Edge effects on an epiphytic lichen in fragmented forests. Conservation Biology 12:1307-1317.

Ghalambor, C. K. And T. E. Martin. 1999. Red-breasted Nuthatch (Sitta canadensis). The birds of North America. Number 459.

GonZalez-Abraham, C., V. C. RAdeloff, R. B. HAMmer, T. J. Hawbaker, S. I. STEWART, AND M. K. Clayton. 2006. Building patterns and landscape fragmentation in northern Wisconsin, USA. Landscape Ecology 22: 217-230.

Great Lakes Information Network. 2005. Great Lakes Information Network. <www.great-lakes.net> 12 October 2005. Ann Arbor, Michigan, USA.

Gustafson, E. J., R. B. HAMmer, V. C. RAdelofF, AND R. S. PotTs. 2005. The relationship between environmental amenities and changing human settlement patterns between 1980 and 2000 in the midwestern USA. Landscape Ecology 20:773-789.

Hostetler, M. AND C. S. Holling. 2000. Detecting the scales at which birds respond to structure in urban landscapes. Urban Ecosystems 4:25-54.

Lee, M., L. Fahrig, K. Freemark, And D. J. Currie. 2002. Importance of patch scale vs. landscape scale on selected forest birds. Oikos 96:110-118.

LEVIN, S. A. 1992. The problem of pattern and scale in ecology: the Robert H. MacArthur Award Lecture. Ecology 73:1943-1967.

Lindsay, A. R., S. S. Gillum, And M. W. Meyer. 2002. Influence of lakeshore development on breeding bird communities in a mixed northern forest. Biological Conservation 107:1-11.

LONG, L. AND A. NuCCI. 1997. The 'clean break' revisited: is U.S. population again deconcentrating? Environmental Planning 29:1355-1366. 
MagurRAn, A. E. 2004. Measuring biological diversity. Blackwell Publishing, Oxford, United Kingdom.

MANAROLla, M. T. 2005. Breeding bird community structure in developed and undeveloped areas along the Lake Superior shoreline. Thesis. Michigan Technological University, Houghton, USA.

Mills, S. G., J. B. Dunning, And J. M. BAtes. 1989. Effects of urbanization on breeding bird community structure in southwestern desert habitats. Condor 91: 416-428.

Moldenhauer, R. R. AND D. J. Regelski. 1996. Northern Parula (Parula americana). The birds of North America. Number 215.

Naiman, R. J., H. DécAmps, And M. Pollock. 1993. The role of riparian corridors in maintaining regional biodiversity. Ecological Applications 3:209-212.

Noon, B. R. 1981. Techniques for sampling avian habitats. USDA, Forest Service, Technical Report RM-87. Rocky Mountain Forest and Range Experiment Station, Fort Collins, Colorado, USA.

ORR, B. 1997. Land use change on Michigan's Lake Superior shoreline: integrating land tenure and land cover type data. Journal for Great Lakes Research 23:328-338.

Quinn, G. P. And M. J. KeOUgh. 2002. Experimental design and data analysis for biologists. Cambridge University Press, Cambridge, United Kingdom.

Radeloff, V. C., R. B. Hammer, P. R. Voss, A. E. Hagen, D. R. Field, and D. J. Mladenoff. 2001. Human demographic trends and landscape level forest management in the northwest Wisconsin pine barrens. Forest Science 47:229-241.

Reynolds, R. T., J. M. ScotT, AND R. A. Nussbaum. 1980. A variable circular-plot method for estimating bird numbers. Condor 82:309-313.

Riffell, S. K., B. E. KeAs, And T. M. Burton. 2001. Area and habitat relationships of birds in Great Lakes coastal wet meadows. Wetlands 21:492-507.

RobiIns, C. S. 1981. Bird activity levels related to weather. Studies in Avian Biology 6:301-310.

RotTenborn, S. C. 1999. Predicting the impacts of urbanization on riparian bird communities. Biological Conservation 88:289-299.

SAAB, V. 1999. Importance of spatial scale to habitat use by breeding birds in riparian forests: a hierarchical analysis. Ecological Applications 9:135-151.

Sallabanks, R., J. R. Walters, and J. A. Collazo. 2000. Breeding bird abundance in bottomland hardwood forests: habitat, edge, and patch size effects. Condor 102:748-758.

Schnaiberg, J., J. Riera, M. J. Turner, And P. R. Voss. 2002. Explaining human settlement patterns in a recreational lake district: Vilas County, Wisconsin, USA. Environmental Management 30:24-34.

Smith, R., M. J. Hamas, and M. E. Dallman. 1998. Spatial variation in foraging of the Black-throated Green Warbler along the shoreline of northern Lake Huron. Condor 100:474-484.

Sutherland, W. J., I. Newton, AND R. E. Green. 2004. Bird ecology and conservation. Oxford University Press Inc. New York, USA.

SwEnSON, J. J. AND J. FRANKLIN. 2000. The effects of future urban development on habitat fragmentation in the Santa Monica Mountains. Landscape Ecology 15:713730 .

Theobald, D. M., J. R. Miller, and N. T. Hobbs. 1997. Estimating the cumulative effects of development on wildlife habitat. Landscape and Urban Planning 39:25-36.

Vale, T. R. AND G. R. Vale. 1976. Suburban bird populations in west-central California. Journal of Biogeography 3:157-165.

WeAther Underground CoRPORATION. 2005. History for Houghton, Michigan. Weather Underground Corporation. 1 October 2005. <www.weatherunderground.com>

Wiens, J. A. 1989. The ecology of bird communities. Cambridge University Press, Cambridge, United Kingdom.

Willson, M. F. 1974. Avian community organization and habitat structure. Ecology 55:1017-1029.

Woodford, J. E. AND M. W. MEYer. 2003. Impact of lakeshore development on green frog abundance. Biological Conservation 110:277-284. 